\title{
Quadrature Decomposition of Optical Fields using two Orthogonal Phase Sensitive Amplifiers
}

\author{
F. Parmigiani, R. Slavík, G. Hesketh, P. Petropoulos, D. J. Richardson \\ Optoelectronics Research Centre, University of Southampton, Southampton, SO17 1BJ, UK \\ (frp@orc.soton.ac.uk)
}

\begin{abstract}
We propose a new technique to optically process coherent signals by simultaneously extracting their two (I and Q) quadrature components into two orthogonal polarizations at the same frequency. Two possible implementations are demonstrated.
\end{abstract}

\section{Introduction}

New optical approaches to fully process signals modulated using the newly emerged and spectrally efficient modulation formats are strongly needed. In these formats, the dimension space is increased by modulating the information independently in the two orthogonal optical field components (in-phase (I) and quadrature (Q)). For detection, a coherent receiver is commonly used, producing two independent data streams, and the original data are then extracted from these data streams using Digital Signal Processing (DSP). Although powerful and efficient, this approach requires optical-to-electrical (O-E) conversion and it may not be the optimal choice when the processing of the signals is desired solely in the optical domain. In this scenario, the capability to optically decompose the incoming signal into the I and Q components would be highly desired. To that end, M. Gao et al. ${ }^{1}$ demonstrated demultiplexing of a quadrature phase shift keying (QPSK) signal into two binary phase shift keying (BPSK) signals, i.e. the two quadrature components. In their method, it was possible to recover one quadrature at a time (either I or $Q$ ) by controlling the relative phases between the pump and signal waves in a single phase sensitive amplifier (PSA). Using a different PSAbased configuration a similar demultiplexing experiment was demonstrated ${ }^{2-3}$ which simultaneously separated the two quadratures of the signal by converting them to different wavelengths. While this scheme allowed for simultaneous decomposition of the signal into the two quadratures, it required four phaselocked pumps and, more importantly, it would impose challenging requirements if the two quadratures at different wavelengths needed to be recombined back into a single one.

Here, we experimentally demonstrate how our newly proposed PSA configuration, which we referred to as polarization assisted (PA) $\mathrm{PSA}^{4}$, can be adapted to simultaneously decompose the I and $Q$ components of a signal on the same wavelength but at two orthogonal polarization

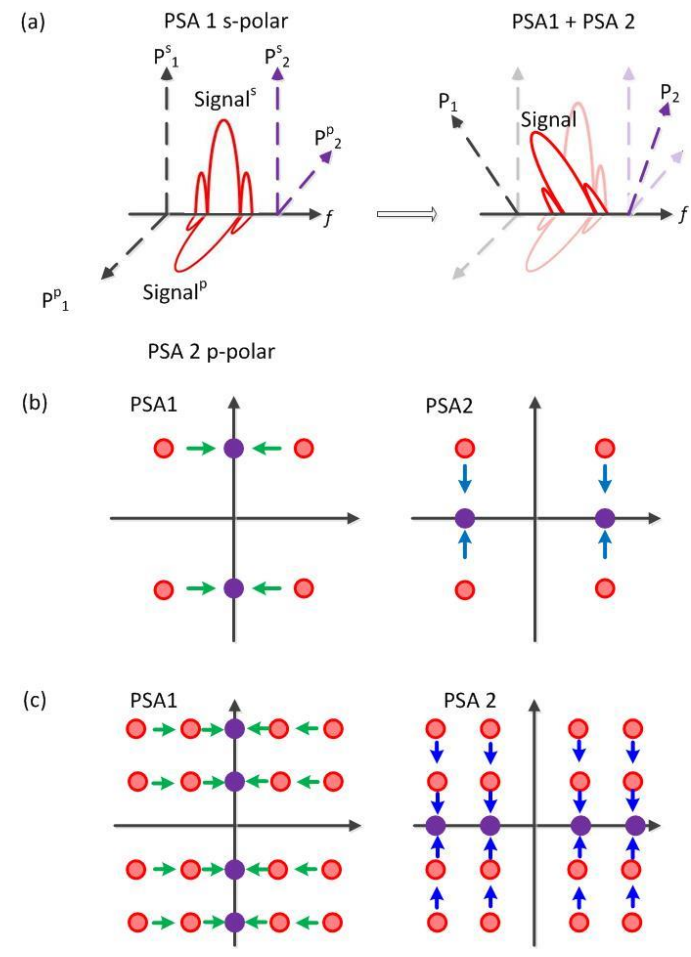

Fig. 1: Operation principle sketch (a) and two examples of optical field quadrature decomposition for QPSK (b) or 16 QAM (c).

axes. This allows us to independently access the I and $Q$ components and simply recombine them back into one data stream, should this be required. Two examples of this optical processor are reported: i) simultaneous demodulation of a QPSK signal into its I and Q constituents and ii) regeneration of a QPSK signal, achieved by regenerating in parallel (and in the same medium) the two separate BPSK streams, i.e. the in-phase and quadrature field decompositions of the original signal, before coherently recombining them.

\section{Operating Principle}

The operating principle is sketched in Fig. 1 (a). It is based on the generation of two degenerate dual-pump scalar PSAs operating simultaneously along two polarization axes, $s$ and $\mathrm{p}$, with a $\pi / 2$ phase shift between them. In 


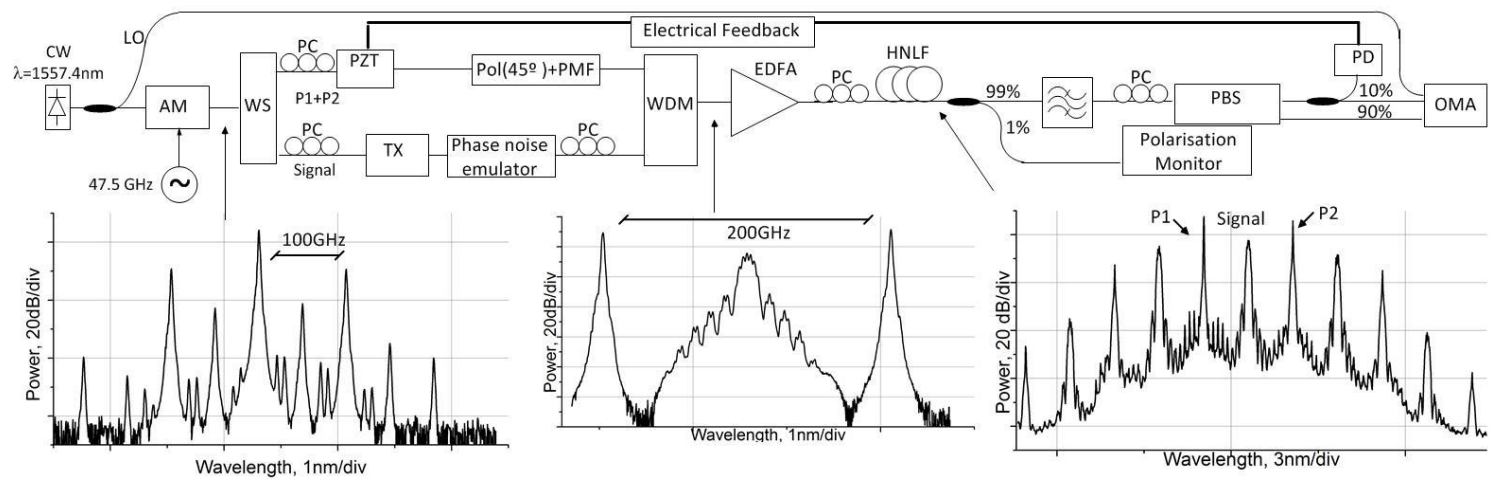

Fig. 2: Experimental set-up. Inset figures: Spectral traces at different point of the system. From the left: initial optical comb before and after the HNLF. LO: local oscillator, TX: transmitter, Pol: polarizer, PC: polarization controller, EDFA: erbium doped amplifier, PD: photodiode.

our implementation the $s$ and $p$ components of Pump 1 (P1) are in phase, as are the $s$ and $p$ components of the signal, while the $p$ component of Pump 2 (P2) has a $\pi$ phase shift relative to its $s$ component, hence $\mathrm{P} 2$ has the opposite sign to $\mathrm{P} 1$ and the signal in the $\mathrm{p}$ polarization, as shown in the left of Fig.1 (a). The vector addition of these components is shown on the right of Fig.1 (a) (bold lines), with $\mathrm{P} 1$ and the signal co-polarized and P2 orthogonally polarized relative to them. As a consequence, the gain region of the PSA along the $p$ - polarization (PSA2) will be shifted by $\pi / 2$ with respect to the PSA along the s- polarization (PSA1). By placing a polarization beam splitter (PBS) at the output of the nonlinear medium with its polarization axes aligned to the $s$ - and $p$ polarizations, i.e. aligned to the two PSAs (at $\pm 45^{\circ}$ relative to the input signal polarization), it is possible to simultaneously extract the two PSA outputs. These will contain the decomposed orthogonal quadratures of the signal provided that the two PSAs exhibit suitably high phase sensitive extinction ratios (PSERs - defined as the difference between the maximum PS gain and the maximum PS de-amplification).

We have previously demonstrated high PSERs using PAPSA at various (low) power levels simply by polarization filtering (through a polarizer) the PSA output at an angle $\alpha$ relative to the original signal polarization axis ${ }^{4}$. While in principle, a second polarizer at an angle of $-\alpha$ could be used to yield the orthogonal component of the signal, allowing operation at low power levels, it is easier to consider high power levels in order to achieve $\alpha=\pi / 4$, thus a single polarization beam splitter (PBS) can be used to extract simultaneously the $\mathrm{I}$ and $\mathrm{Q}$ components of the signal at its two outputs. Fig.1 (b) and Fig.1 (c) sketch two examples of optical field quadrature decomposition for QPSK and 16 quadrature amplitude modulation (QAM) signals, respectively, via PSA1 and PSA2.

Furthermore, if a QPSK signal is considered at the input and if saturation is achieved for the two PSAs, then it is possible to effectively recombine the output of the two PSAs (simply by using a PBS aligned (orthogonal) to the original signal, i.e. at $\pm \pi / 4$ with respect to the s-polarization axis). Each of the two PSA outputs represents an individually regenerated BPSK stream ${ }^{5}$, thereby resulting in a regenerated QPSK signal after recombination at the PBS output. This is conceptually similar to what had been proposed in [6]. Here, however, both the regeneration and the recombination are achieved within the same fiber path, which has significant practical implications with respect to the relative phaselocking between the various waves.

\section{Experimental Set-up and Results}

Our experimental set-up is shown in Fig. 2. An overdriven amplitude modulator (AM) was used to modulate a $17 \mathrm{dBm}, 1557.4 \mathrm{~nm}$ continuous wave $(\mathrm{CW})$ laser to produce an optical frequency comb with $50 \mathrm{GHz}$ spacing, see inset to Fig. 2. The comb was filtered and demultiplexed using a programmable filter (Waveshaper, WS) to separately select three of its lines acting as the phase-locked signal (directed to one path) and two pump waves P1 and P2 (directed to another path). To obtain an orthogonally polarized $\mathrm{P} 2$ with respect to $\mathrm{P} 1$, the initially linearly co-polarized pumps, spaced by $200 \mathrm{GHz}$, were aligned at $45^{\circ}$ with respect to the primary polarization axis of a carefully chosen short length (about $2 \mathrm{~m}$ ) of polarization maintaining fiber (PMF). The signal was QPSKmodulated at 10 Gbaud. In the experiment targeting regeneration, the signal was sent through a noise additive module, which comprised a single tone sinewave at $\sim 1.1 \mathrm{GHz}$ that drove an optical phase modulator through which the signal was passed, in order to 


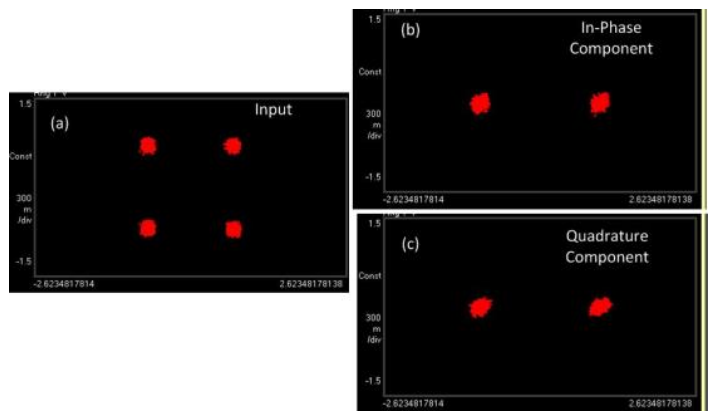

Fig. 3: Field decomposition of a QPSK signal.

emulate the effects of phase noise. Following this, the signal was combined with the pumps with the desired polarization and all the waves were amplified in an EDFA up to a total power of $\sim 29 \mathrm{dBm}$ at the HNLF input. The parameters of the $302 \mathrm{~m}$-long germanium-silicate dispersionshifted strained fibre are as follows: nonlinear coefficient of $11.6(\mathrm{~W} \cdot \mathrm{km})^{-1}$, dispersion slope of $0.018 \mathrm{ps} /\left(\mathrm{nm}^{2} \cdot \mathrm{km}\right)$ and zero dispersion wavelength after straining of $1555 \mathrm{~nm}$. Examples of the spectra at the input/output of the HNLF are shown inset to Fig. 2. A portion of the beams was used to monitor the relative polarization of the signal and the pumps. At the output of the HNLF, a PBS allowed selection of the desired polarization axis (/axes) for either the separation or recombination of the two individual PSAs. The signal was then assessed using an Agilent Optical Modulation Analyzer (OMA). Slow thermo-acoustic relative phase drifts were suppressed by monitoring the signal power at the system output and controlling a piezo-electric transducer (PZT) located at the path of the two pumps.

Firstly, the proposed scheme was configured to demodulate the QPSK signal (error vector magnitude, EVM, of $7.4 \%$ root mean square, rms), into its two quadrature components (see Fig.3 (a)). For this case, the PBS was aligned to the two PSAs and the results are shown in Fig.3 (b,c) for the I and Q respectively, achieving EVMs of less than $8.5 \% \mathrm{rms}$ for both components.

Secondly, the proposed scheme was configured to perform phase regeneration of the QPSK signal. In this case, the PSAs were operating in saturation and the polarizer was aligned (orthogonal) to the incoming signal. The system was first tested without any phase noise added to the signal, see Fig. 4 (a) and Fig. 4 (b), showing a small degradation at the system output. When noise was added, see Fig. 4 (c), to achieve an EVM, magnitude and phase errors of $\sim 20 \% \mathrm{rms}, 6 \% \mathrm{rms}$ and $11 \mathrm{deg}$ respectively, the regenerator improved the signal performance, leading to EVM, magnitude and phase errors of

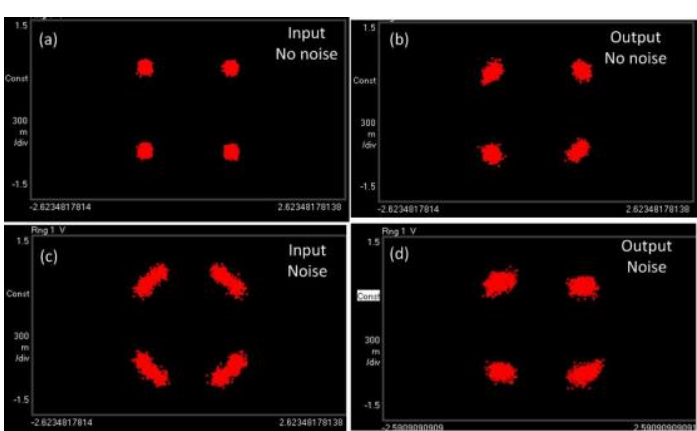

Fig. 4: Measured constellation diagrams at the input and output of the scheme when it is implemented as a regenerator with and without noise added to the incoming QPSK signal.

$14 \% \mathrm{rms}, 8 \% \mathrm{rms}$, and 6 deg respectively, i.e. reducing the initial phase noise to almost a half.

\section{Conclusions}

We have proposed a new technique to optically process coherent signals by simultaneously extracting the two ( $I$ and $Q$ ) quadrature components into two orthogonal polarizations at the same frequency, by using two identical and orthogonal PSAs in a single HNLF. Two possible examples were experimentally demonstrated to highlight the potential of the scheme: decomposition on the two quadratures of a QPSK signal and regeneration of a QPSK signal with a phase noise reduction of almost half (from 11 degrees to 6 degrees).

\section{Acknowledgements}

F. P. gratefully acknowledges the support from the Royal Academy of Engineering/EPSRC through a University Research Fellowship. This research is sponsored by EPSRC grant EP/I01196X, The Photonics Hyperhighway. We thank OFS for supplying the HNLF used in this experiment.

\section{References}

[1] M. Gao et al., "Low-penalty Phase De-multiplexing of QPSK Signal by Dual-pump Phase Sensitive Amplifiers", ECOC, We.3.A.5 (2013).

[2] R.P. Webb, M. Power, and R.J. Manning, "Phasesensitive frequency conversion of quadrature modulated signals," Opt. Express 21, 12713-12727 (2013).

[3] F. Da Ros, et al., "QPSK-to-2×BPSK wavelength and modulation format conversion through phase-sensitive four-wave mixing in a highly nonlinear optical fiber", Optics Express 21 (23), pp. 28743-28750 (2013).

[4] F. Parmigiani, et al. "Novel Polarization-assisted Phase Sensitive Optical Signal Processor Requiring Low Nonlinear Phase Shifts", STu2I.4 CLEO US 2014.

[5] R. Slavik, et al., "All-optical phase and amplitude regenerator for next-generation telecommunications systems," Nat. Phot., vol. 4, pp. 690-695, Oct. 2010.

[6] Z. Zheng, et al., "All-optical regeneration of DQPSK/QPSK signals based on phase-sensitive amplification," Optics Communications, vol. 281, pp. 2755-2759, 2008. 